\title{
Situationally Aware Mobile Devices for Overcoming Situational Impairments ${ }^{*}$
}

\author{
Jacob O. Wobbrock \\ The Information School | DUB Group, University of Washington, Seattle, WA, USA, wobbrock@uw.edu \\ * This article is based upon a forthcoming book chapter by the author [48]. There, interested readers will find a large \\ categorized survey of related work on situationally induced impairments and disabilities.
}

\begin{abstract}
Situationally induced impairments and disabilities (SIIDs) can compromise people's use of mobile devices. Factors like walking, divided attention, cold temperatures, low light levels, glare, inebriation, fear, loud noises, or rainwater can make using a device in off-desktop environments challenging and even unsafe. Unfortunately, today's mobile devices know almost nothing about their users' situations, contexts, or environments, instead employing many of the same interaction concepts found on desktop systems from the 1980s. This article presents a decade's worth of work from 2008-2018 in making mobile devices more situationally aware and capable of improving interaction for users experiencing SIIDs. Also presented is a categorized list of factors that can cause SIIDs, and a two-dimensional space for characterizing impairments. Seven specific research projects are summarized, which variously address walking, hand grips, divided attention, distraction, inebriation, and rainwater interference. A "sense-model-adapt" design pattern emerges from many of these projects for addressing SIIDs. Taken together, these projects demonstrate how mobile devices can be made more situationally aware and better capable of supporting users' interactions on-the-go.
\end{abstract}

\section{CCS CONCEPTS}

- Human-centered computing Touch screens • Human-centered computing Mobile computing

- Human-centered computing Smartphones • Human-centered computing Mobile phones

- Human-centered computing Text input • Human-centered computing Ubiquitous computing • Humancentered computing $\sim$ Accessibility theory, concepts and paradigms $\bullet$ Human-centered computing $\sim$ Gestural input $•$ Human-centered computing Ubiquitous and mobile computing design and evaluation methods - Human-centered computing Empirical studies in ubiquitous and mobile computing

\section{KEYWORDS}

Situationally induced impairments and disabilities; smartphones; walking user interfaces; mobile humancomputer interaction; sensors; user modeling; social model of disability; accessibility for all.

\section{Introduction}

Computer users of today interact with mobile computing systems in situations that would be unrecognizable to computer users of the early desktop era of the 1980s. Users of those early interactive systems worked in staid office environments on computers sitting on stable surfaces, with ample lighting, controlled temperatures, and minimal noise or distraction. They operated their systems with two hands without outside

Permission to make digital or hard copies of all or part of this work for personal or classroom use is granted without fee provided that copies are not made or distributed for profit or commercial advantage and that copies bear this notice and the full citation on the first page. Copyrights for components of this work owned by others than ACM must be honored. Abstracting with credit is permitted. To copy otherwise, or republish, to post on servers or to redistribute to lists, requires prior specific permission and/or a fee. Request permissions from Permissions@acm.org.

EICS '19, June 18-21, 2019, Valencia, Spain

(C) 2019 Association for Computing Machinery.

ACM ISBN 978-1-4503-6745-5/19/06 ..\$15.00

https://doi.org/10.1145/3319499.3330292 
forces acting upon their bodies while seated comfortably in physically safe and enclosed environments, protected from the elements. By contrast, computer users of today are likely to be using a touch screen device, perhaps while on-the-go, perhaps while outside, perhaps while surrounded by attention-grabbers like people, traffic lights, curbs, and street signs. They might be trying to interact while carrying luggage, wearing gloves, or wiping rainwater from their screens. They might be trying to walk on uneven terrain, or to move up or down stairways, over broken sidewalks, or amidst advancing vehicles. They might have stiff fingers due to cold temperatures, or sweaty hands due to high humidity. They might be in the dark, or in the blazing glare of intense sunlight.

The era of mobile and ubiquitous computing has enabled computer users of today to break out of the office and into the world, bringing the benefits of computing into almost every aspect of modern life. But these benefits come at a cost. Our mobile devices today know almost nothing of our challenging new contexts of use, and offer even less to us by way of help or support for interaction. Most seriously, interacting with mobile devices while on-the-go can risk a person's safety, or the safety of others.

The costs of interacting while mobile are increasingly coming into view. These costs come in (at least) cognitive, perceptual, motor, and social forms. For example, studies show that interacting on a mobile device while walking reduces reading speed [28], reduces motor accuracy [7,20,21,37], divides attention [5,33], and reduces one's ability to maneuver and avoid obstacles [44]. Walking itself is also negatively affected: walking speed slows by $30-40 \%$ when interacting while on-the-go [2,3,20,26,37,38]. More concerning, studies show that 1 in 3 Americans in crosswalks are focused on their smartphones [42]. The U.S. Federal Communications Commission (FCC) cites a statistic from the National Highway Traffic Safety Administration (NHTSA), stating that "[d]uring daylight hours across America, approximately 481,000 drivers are using cell phones while driving." " The FCC also reports an NHTSA statistic that "3,166 people were killed by distracted driving in 2017 alone, 8.5 percent of total fatalities for the year." Whether it is walking with a smartphone, operating a tablet with one hand, or driving and texting, our mobile devices generally have little idea of the specific situations in which we interact with them. Although smartphones have an indication of where they are on Earth via global positioning systems (GPS), and although some phones when linked into automotive computing systems force their users to indicate they are not driving, our mobile devices have strikingly little awareness of what is going on around them. This state of affairs might be acceptable for desktop computers, but mobile devices go with us everywhere and are involved in everything-yet without any appreciation for how they degrade our abilities to think, perceive, act, and communicate with others.

So how have we, as a society, dealt with these challenges? Approaches have generally focused on posting warnings, issuing fines, redesigning environments, or altering users - seemingly anything but making our mobile devices more situationally aware and capable of better supporting our abilities to interact with them, others, and the world around us. For example, in Hayward, California, municipal street signs warn pedestrians to first cross the street and only then update Facebook [1]. In Stockholm, Sweden, warning signs were thought to be more effective for drivers than walkers, so signs depicting silhouettes of heads-down pedestrians glued to their smartphones caution drivers not to run them over [13]. Beyond warning signs, the Utah Transit Authority imposes a $\$ 50$ fine for texting while walking near light rail tracks [46]. Similarly, Honolulu, Hawaii issues $\$ 35$ fines to people for "viewing their electronic devices while crossing streets in the city and surrounding county" [27]. But warnings and fines only do so much, so some cities have changed the design of their built environments. For example, Augsburg, Germany and Bodegraven, Netherlands have embedded crosswalk signals into their sidewalks so that pedestrians looking down at their phones might halt before stepping into traffic [27,31]. The Chinese city of Chongqing created separate lanes for walkers using their smartphones and for walkers promising to refrain [30]. A neighborhood in London, England even tried putting pads on their lamp posts to prevent injuries for texting walkers [34]. Finally, some approaches alter users themselves to accommodate mobile devices in certain environments, such as wearing gloves with capacitive-sensing fingertips in cold weather, or donning noise-canceling headphones in clamorous environments. And yet, none of these approaches puts the onus on the mobile devices themselves to become more situationally aware, responsive to environments, and better capable of supporting users' interactions.

${ }^{1}$ https://www.fcc.gov/consumers/guides/dangers-texting-while-driving 
This article summarizes work from 2008 - 2018 by the author and his collaborators devoted to making mobile devices more situationally aware in service of improving interaction for users in off-desktop environments while on-the-go. The specific viewpoint adopted in this work is that situations, contexts, and environments impair users' abilities, causing situationally induced impairments and disabilities (SIIDs) [39,40]. As Sears and Young [40], who first conceptualized SIIDs, wrote:

Both the environment in which an individual is working and the current context (e.g., the activities in which the person is engaged) can contribute to the existence of impairments, disabilities, and handicaps (p.488).

The remainder of this article further develops the position that mobile human-computer interaction can be improved by enabling mobile devices to address SIIDs. By making mobile devices more situationally aware, they can better support their users' situated abilities.

\title{
2 Ability, Disability and Environment: An Evolving View
}

The approach to improving mobile human-computer interaction (HCI) by enabling mobile devices to address situationally induced impairments and disabilities (SIIDs) raises some questions about the notions of ability and disability. It is clear that situations, contexts, and environments ${ }^{2}$ can negatively affect users' abilities, and many within HCI and accessible computing have observed a connection to disability $[9,29,39,40,45,47]$. But any honest consideration of SIIDs must acknowledge that they are and should be controversial. Are such "disabilities" even disabilities at all? What might be the harms to people with sensory, physical, or cognitive disabilities by employing a concept like SIIDs?

The point must be made emphatically that in this view, there can be no equating of the lived experiences of people with disabilities to those of people experiencing SIIDs. As I have written elsewhere [48]:

\begin{abstract}
One might argue that calling a "disability" that which can be alleviated by a change in circumstances diminishes the lived experiences of those with lifelong disabilities. A person experiencing a situational disability suffers neither the sting of stigma nor the exile of exclusion. Modern social scientists acknowledge that disability is as much a cultural identifier as it is a personal ascription [22,35,41], and nondisabled people experiencing a situational disability take no part in, and make no contribution to, a “disability culture." In fact, neither a person experiencing a situational disability, nor anyone observing him or her, regards that person as having a disability at all. No accommodations are required; no laws must be enacted; no rights must be protected or enshrined. Perhaps, therefore, the notion of SIIDs is not only wrong, but also misguided and offensive.
\end{abstract}

Nonetheless, there are three reasons why SIIDs are a useful framing - a framing that, rather than detracting from the experiences of people with disabilities, can serve to highlight the relevance of accessibility to everyone.

The first reason is that the notion of "disability" has, fortunately, evolved since the 1970s from the medical model, in which the disability resides purely in the person, to the social model, which specifically calls out environmental factors as contributing to disabilities [51]. Under this view, a person in a wheelchair facing a flight of concrete stairs is not disabled solely because of his inability to walk and his reliance upon the chair; in fact, the concrete stairs are an inextricable contributing factor, and they should be changed (e.g., augmented with a ramp) rather than the person. But if we are to acknowledge these environmental factors in contributing to conventional disabilities, then we must also do so for people without sensory, physical, or cognitive impairments. Put another way, the component of disability to which environmental factors contribute applies to everyone, and thus everyone has an investment in making the world a more accessible place, even if only on selfish grounds.

\footnotetext{
${ }^{2}$ Readers wanting an account of the differences between situations, contexts, and environments are directed to elaborations elsewhere [48].
} 
The second reason is that from a design perspective, the claim is not that people with disabilities and people experiencing SIIDs are somehow "the same," but rather, that the designs that might serve their needs are the same. A mobile user interface that enables one-handed use could similarly help a person with one arm and a person carrying an infant or a bag of groceries. Thus, the focus is no longer on what a person cannot do, but on what a person can do given their situated abilities $[4,8,29]$. This perspective shift lies at the core of ability-based design [49,50], a design perspective that scrutinizes technologies for their underlying ability assumptions, and places the onus on technologies to better accommodate their users' abilities rather than on users to adapt themselves to their technologies.

The third reason is that SIIDs have real, even life or death, consequences because, although they are temporary, people's abilities are significantly diminished by them. In this view, it is not that conventional disabilities are serious and SIIDs are a flippant version of the same. On the contrary, people die every day because of the effects of SIIDs. The American Centers for Disease Control and Prevention estimates that 9 people are killed and 1000 people are injured every day in incidents involving a distracted driver. ${ }^{3}$ And pedestrians sometimes referred to as "walking zombies" are injured regularly by colliding with other people, objects, and vehicles $[6,15,36]$. Thus, disability is a serious matter in all its forms, which should not be mistaken for an argument that all forms of disability are therefore comparable.

Pioneers in accessible computing have highlighted the link between designing for people with disabilities and designing for people in disabling situations for 25 years. For example, in 1995, Allen F. Newell edited a volume entitled Extra-Ordinary Human-Computer Interaction in which he wrote a chapter with a section called "People are handicapped by their environments" [29]. In that section, he described a soldier on a battlefield:

He or she can be blinded by smoke, be deafened by gunfire, be mobility impaired by being up to the waist in mud, have poor tactile sensitivity and dexterity because of wearing a chemical warfare suit, and be cognitively impaired because of being scared stiff of being killed - and this is before the solider is wounded! If one were to measure the effective abilities of a person in such an environment, they would be poor enough over a number of dimensions for him or her to be classified as severely disabled in a more normal environment ( $p .9)$.

Newell's general argument is that everyone has abilities and limits to those abilities; some abilities are ordinary and some are extra-ordinary. Regardless, situations, contexts, and environments put limitssometimes severe ones - on the abilities of all people.

Another pioneer in accessible computing, Gregg C. Vanderheiden, made similar observations, although his were focused on systems design. As he wrote in 1997 [45]:

If we design systems which are truly ubiquitous and nomadic; that we can use whether we are walking down the hall, driving the car, sitting at our workstation, or sitting in a meeting; that we can use when we're under stress or distracted; and that make it easy for us to locate and use new services-we will have created systems which are accessible to almost anyone with a physical or sensory disability (p. 1439).

In the above quote, Vanderheiden is arguing that similar technology designs can serve different people, those with disabilities and those experiencing SIIDs. He further articulated:

[D]ifferent environments will put constraints on the type of physical and sensory input and output techniques that will work (e.g., it is difficult to use a keyboard when walking; it is difficult and dangerous to use visual displays when driving a car; and speech input and output, which work great in a car, may not be usable in a shared environment, in a noisy mall, in the midst of a meeting, or while in the library). ... [M] ost all of the

\footnotetext{
${ }^{3}$ https://www.cdc.gov/motorvehiclesafety/distracted_driving/index.html
} 
issues around providing access to people with disabilities will be addressed if we simply

address the issues raised by [this] "range of environments" (p. 1440).

Indeed, the "range of environments" that Vanderheiden recognized in 1997 has only become greater today with mobile devices permeating every aspect of professional and personal life. But unfortunately, our devices are still almost as situationally unaware as they were back then-a shortcoming that the research reported here has tried to address.

\section{Defining the Space of Situational Impairments}

Section 4 presents examples of specific research projects that have demonstrated mobile devices' enhanced situational awareness for overcoming situationally induced impairments and disabilities (SIIDs). But first, in this section, it is useful to formulate a categorization of the factors that cause SIIDs. It is also useful to formulate a two-dimensional space to characterize these factors. These organizing schemes can enable us to think carefully about where to place our research and design efforts.

Table 1 shows a categorization of situational factors, broken into the following six themes: Behavioral, Environmental, Attentional, Affective, Social, and Technological. The table makes clear just how few situational factors our mobile devices currently can detect. For an expanded version of this table that includes extensive references to prior work, the reader is directed elsewhere [48].

Table 2 offers a two-dimensional characterization of impairments. It characterizes impairments along two dimensions: location and duration. Location refers to the source of the impairment, namely whether it comes from within a person ("intrinsic"), from outside a person in the environment ("extrinsic"), or from a combination of both ("mixed"). Duration refers to how long-lived the impairment is, ranging from seconds or minutes to years or decades. Most SIIDs are located outside a person and are short-lived. Many sensory, physical, or cognitive impairments, by contrast, are located within a person and are long-lived.

\section{Making Mobile Devices More Situationally Aware: A Decade of Projects}

Let us recap the major positions asserted in this article thus far: (1) today's mobile devices are used in a variety of off-desktop situations, contexts, and environments; (2) many of these situations, contexts, and environments can cause situationally induced impairments and disabilities (SIIDs), negatively impacting users' abilities to interact with their devices, other people, and the world around them; (3) SIIDs can be serious, even life-threatening, especially when involving automobiles; (4) designing for users experiencing SIIDs would also likely create more accessible designs for people with disabilities, and vice versa; and (5) our mobile devices are generally unaware of their contexts of use, and making them more situationally aware could help address SIIDs.

Over the last decade, my collaborators and I have pursued a variety of projects in mobile humancomputer interaction devoted to ameliorating SIIDs by making mobile devices more situationally aware. In each project, no additional or custom sensors were added to the mobile device beyond its built-in commercial sensors. This choice was made to show the potential for software to address SIIDs, rather than requiring custom hardware that is not widely available. The subsections that follow give a brief overview of seven projects from $2008-2018$. Interested readers are directed to the original papers for more detail.

\subsection{Walking User Interfaces (2008)}

In 2008, Kane et al. [18] coined the phrase "walking user interface," or "WUI" for short, to refer to an interface that adapts its presentation based on whether or not the user is walking. In our prototype of a music player on an ultra-mobile PC (see Figure 1), when the user was stationary, the music list was high-density, showing more items with smaller fonts and buttons. When the user was walking, the list became low-density, showing fewer items with fonts and buttons that were 2-3 times bigger, but that required more scrolling (albeit on a larger scrollbar). 
Table 1. Situational factors that can cause SIIDs. This table is derived from prior work [48].

\begin{tabular}{|c|c|}
\hline Category & Factors \\
\hline \multirow[t]{11}{*}{ Behavioral } & Walking \\
\hline & Riding (e.g., in a car, bus, etc.) \\
\hline & Driving \\
\hline & Operating machinery (e.g., in a factory) \\
\hline & Navigating obstacles \\
\hline & Various postures or grips \\
\hline & One-handed use \\
\hline & Hands-busy, hands-free use \\
\hline & Encumbrance (e.g., carrying luggage) \\
\hline & Device out-of-reach \\
\hline & Wearing impeding clothing (e.g., gloves) \\
\hline \multirow[t]{12}{*}{ Environmental } & Vibration \\
\hline & Cold temperatures \\
\hline & Ambient noise \\
\hline & Rainwater \\
\hline & Humidity \\
\hline & Dim light, darkness \\
\hline & Bright light, glare \\
\hline & Coloration \\
\hline & Smoke, fog, smog, haze \\
\hline & Difficult terrain (e.g., ice, mud, stairs) \\
\hline & Confinement, constraining spaces \\
\hline & Extraneous forces (e.g., G-forces) \\
\hline \multirow[t]{7}{*}{ Attentional } & Divided attention \\
\hline & Distraction \\
\hline & Diverted gaze, eyes-busy, eyes-free use \\
\hline & Interruptions \\
\hline & Multitasking \\
\hline & Information overload \\
\hline & High cognitive workload \\
\hline \multirow[t]{6}{*}{ Affective } & Stress, anxiety \\
\hline & Fear \\
\hline & Fatigue, exhaustion \\
\hline & Haste \\
\hline & Elation \\
\hline & Intoxication \\
\hline \multirow[t]{5}{*}{ Social } & Conversation, multiple conversations \\
\hline & Crowds \\
\hline & Social norms or expectations \\
\hline & Laws, policies, or procedures \\
\hline & Privacy or security concerns \\
\hline \multirow[t]{4}{*}{ Technological } & Small output displays (e.g., tiny fonts) \\
\hline & Small input areas (e.g., tiny keys) \\
\hline & Lack of power (e.g., dead battery) \\
\hline & Lack of connectivity (e.g., WiFi outage) \\
\hline
\end{tabular}


Table 2. A space of impairments defined by location and duration and an example of each. This table is reproduced from prior work [48].

\begin{tabular}{|l|l|l|l|}
\cline { 2 - 4 } \multicolumn{1}{c|}{} & \multicolumn{1}{c|}{ Location } \\
\hline Duration & \multicolumn{1}{c|}{$\begin{array}{c}\text { From within } \\
\text { ("intrinsic") }\end{array}$} & \multicolumn{1}{c|}{$\begin{array}{c}\text { From without } \\
\text { ("extrinsic") }\end{array}$} \\
\hline Seconds & $\begin{array}{l}\text { Double vision from } \\
\text { watery eyes }\end{array}$ & $\begin{array}{l}\text { Sneezing from } \\
\text { allergies due to } \\
\text { pollen }\end{array}$ & Loud truck passing \\
\hline Minutes & $\begin{array}{l}\text { Out of breath from } \\
\text { sprinting }\end{array}$ & $\begin{array}{l}\text { Wheelchair user } \\
\text { encountering stairs }\end{array}$ & $\begin{array}{l}\text { Arms full of } \\
\text { groceries }\end{array}$ \\
\hline Hours & Sleeping & Intoxication & $\begin{array}{l}\text { Prisoner's } \\
\text { straightjacket during } \\
\text { prison transfer }\end{array}$ \\
\hline Days & $\begin{array}{l}\text { Soreness from } \\
\text { exercise }\end{array}$ & $\begin{array}{l}\text { Illness from } \\
\text { common cold }\end{array}$ & $\begin{array}{l}\text { Walking over } \\
\text { difficult terrain } \\
\text { while hiking }\end{array}$ \\
\hline $\begin{array}{l}\text { Weeks } \\
\text { more }\end{array}$ & Ankle sprain & $\begin{array}{l}\text { Injured arm in a } \\
\text { hard cast }\end{array}$ & $\begin{array}{l}\text { Solitary } \\
\text { confinement }\end{array}$ \\
\hline Years & $\begin{array}{l}\text { Young children's } \\
\text { development }\end{array}$ & $\begin{array}{l}\text { Seasonal affective } \\
\text { disorder }\end{array}$ & $\begin{array}{l}\text { Darkness in Alaskan } \\
\text { winter }\end{array}$ \\
\hline Addiction & $\begin{array}{l}\text { Incarceration } \\
\text { Muscular dystrophy }\end{array}$ & $\begin{array}{l}\text { Color vision } \\
\text { Insomnia }\end{array}$ & $\begin{array}{l}\text { Heavy water, air, or } \\
\text { soil pollution }\end{array}$ \\
\hline
\end{tabular}




\begin{tabular}{|c|c|}
\hline Cannon & The White Stripes - White St \\
\hline Carbon & Tori Amos - Scarler's Walk \\
\hline Carnival & Natalie Merchant - Tigerlily \\
\hline Changes & David Bowie - Best of Bowie \\
\hline Chemicals & UV Protection - Consumer M \\
\hline China & Tori Amos - Little Earthquake \\
\hline Choke & The Cardigans - First Band Or \\
\hline College & Animal Collective - Sung Tong \\
\hline Communication & The Cardigans - Long Gone B \\
\hline Cotton & The Mountain Goats - We Sh: \\
\hline Cowboy & The Sugarcubes - Life's Too $G$ \\
\hline Crave & Biork - Vespertine \\
\hline Crazy & Tori Amos - Scarle's's Walk \\
\hline Creep & Radiohead - Pablo Honey \\
\hline Crows & The Mountain Goats - Devil ir \\
\hline Cruel & Tori Amos - From The Choir; \\
\hline Crying & Bjork - Debut \\
\hline Curl & Jonathan Coulton - Thing a W \\
\hline Days & David Bowie - Reality \\
\hline \begin{tabular}{|l|l|l|}
41 & II & W \\
\end{tabular} & $\begin{array}{l}\text { mos - Scarlet's Walk } \\
\text { mate }\end{array}$ \\
\hline
\end{tabular}

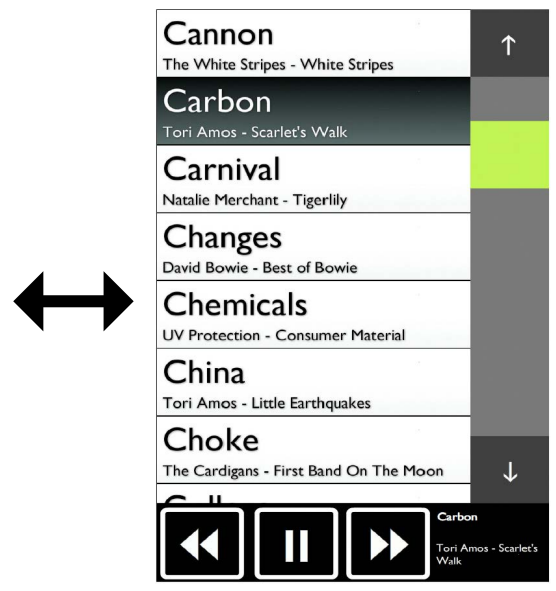

Figure 1. A walking user interface, or WUI. This example shows a music player with songs in a list form. The left interface is shown when the user is standing. The right interface is shown when the user is walking. Figure adapted from prior work [18] with permission from the authors.

A formal experiment with 30 participants and a trained pacer to control outdoor walking speed showed that walking with either static interface increased task completion time by about $18 \%$ compared to standing, but when walking with an adaptive WUI, task time was not detectably different from standing. Put another way, the adaptive WUI avoided the performance penalty incurred by walking.

\subsection{WalkType (2012)}

In 2012, Goel et al. [10] tackled a specific challenge due to walking - that of accurately typing on a smartphone touch screen keyboard. In WalkType, multiple features were combined into various decision tree models that classified keypresses invisibly without any noticeable change to the keyboard (Figure 2). The features included touch properties (down, up, travel, duration), horizontal and vertical direction and displacement of the phone, acceleration of the phone in $(x, y, z)$ to capture vibration, and the phase of the user's gait as inferred by clockwise and counterclockwise rotation of the phone. In addition, touch anchors were used at the very center of each key [14]. The final classification accuracy of the WalkType model was $97.3 \%$ in a 10 -fold cross-validation study based on 47,647 keypresses captured from 16 participants.

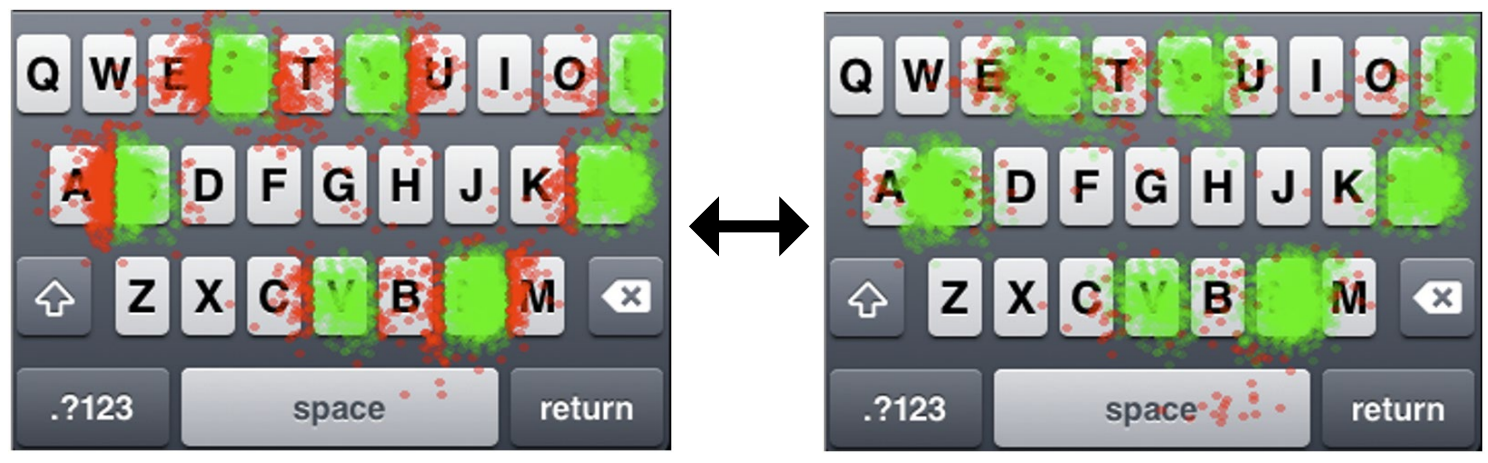

Figure 2. The benefits of the WalkType keyboard. Green dots represent correct keypresses. Red dots represent errors. The left keyboard naively classifies based on key boundaries, while the right keyboard uses WalkType to achieve $45.2 \%$ fewer errors on the same data. (Neither keyboard has any natural language processing features enabled in order to isolate the effects of WalkType on the keypress locations alone.) Figure adapted from prior work [10] with permission from the authors.

To evaluate the interactive benefits of WalkType, we ran a study with a new set of 16 participants who were unaware of any experimental manipulations of the keyboard they were using. Participants were tested 
while both sitting and walking, entering 30 phrases in each condition. In all, we captured 57,663 keypresses. Results showed that, for walking users, WalkType was $12.9 \%$ faster than the de facto keyboard and $45.2 \%$ more accurate, both of which were statistically significant findings. (For sitting users, WalkType was not detectably faster but was mildly more accurate.) In addition, 14 of 16 participants preferred WalkType without having been told anything about it.

\subsection{GripSense (2012)}

While creating WalkType, we asked participants to use two-handed thumb-typing. But an obvious question arose: what if a person used a different grip on the phone? Could there be a way to detect the grip with which someone was holding the phone? That led Goel et al. [12] to create GripSense.

GripSense could detect one- or two-handed grips, distinguish between left- and right-handed use, distinguish between thumb and index-finger use, detect use on a table, and even detect screen pressure without using any force sensors (Figure 3).
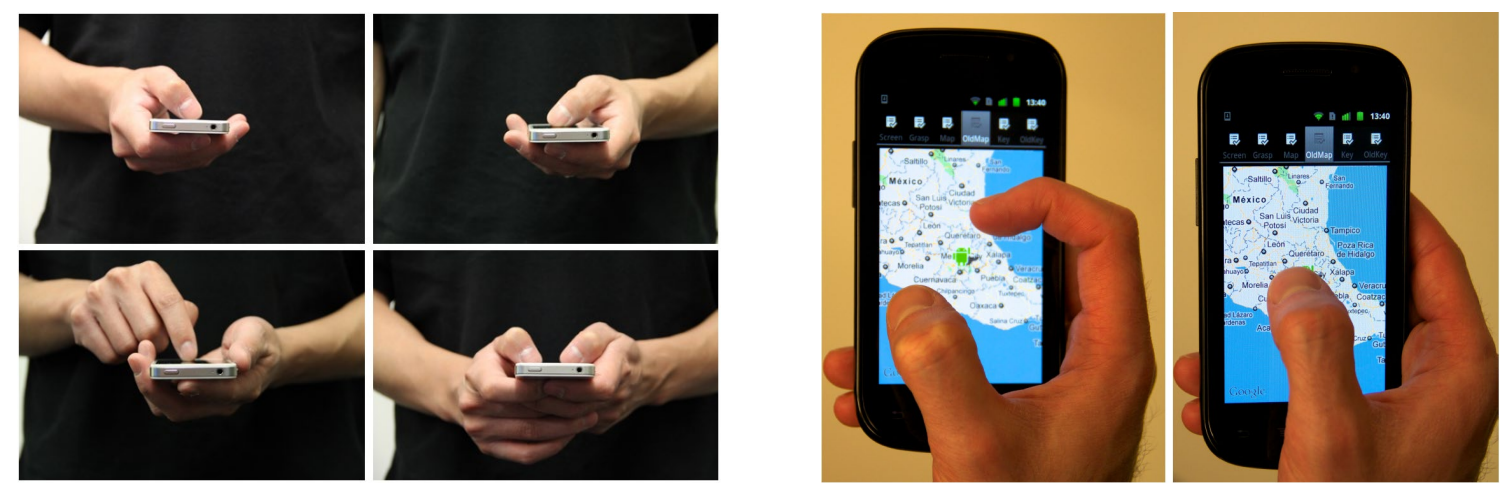

Figure 3. (left) Various hand postures and fingers detected by GripSense. (right) A cumbersome one-handed pinch-to-zoom gesture is replaced by a one-thumb press-to-zoom action with GripSense's pressure-sensing capability. Right figure used from prior work [12] with permission from the authors.

For its grip detection ability, GripSense used a rule-based classifier that considered the rotation of the device via the gyroscope, the size of touches, and the shape of swipe arcs. Its classifier could distinguish inhand use versus on-the-table use with $99.7 \%$ accuracy. It could distinguish among three hand postures (left, right, two-handed) with $84.3 \%$ accuracy within 5.0 taps, and $89.1 \%$ accuracy within 2.4 taps or swipes.

For its pressure detection ability, GripSense used a J48 decision tree classifier that considered the gyroscope, touch size, and touch location. The "trick" for pressure sensing was to pulse the vibration motor of the smartphone when detecting a long-press, and then measure the dampening of the gyroscope signal. Greater thumb pressures dampen the signal amplitude more than lesser pressures. Using thin-film forcesensitive resistors for ground truth, GripSense could distinguish among three pressure levels with $93.5 \%$ accuracy excluding touch size as a feature, and with $95.1 \%$ accuracy including touch size as a feature. With all features, it could distinguish between two pressure levels with $97.9 \%$ accuracy.

\subsection{ContextType (2013)}

With WalkType and GripSense both exhibiting benefits, we sought to further improve smartphone keyboards by making them "grip aware." When typing on a smartphone keyboard with different grips, different error tendencies emerge (see Figure 4). Therefore, Goel et al. [11] created ContextType, a grip-aware keyboard that invisibly changed its underlying keypress classification model based on whichever grip a user was currently employing. ContextType utilized GripSense's posture-sensing and additionally added language modeling to create user-specific touch-and-language models capable of improving text entry accuracy by $20.6 \%$ over such models that did not include grip sensing. Thus, this accuracy improvement represented the benefit of using grip sensing in the models. 


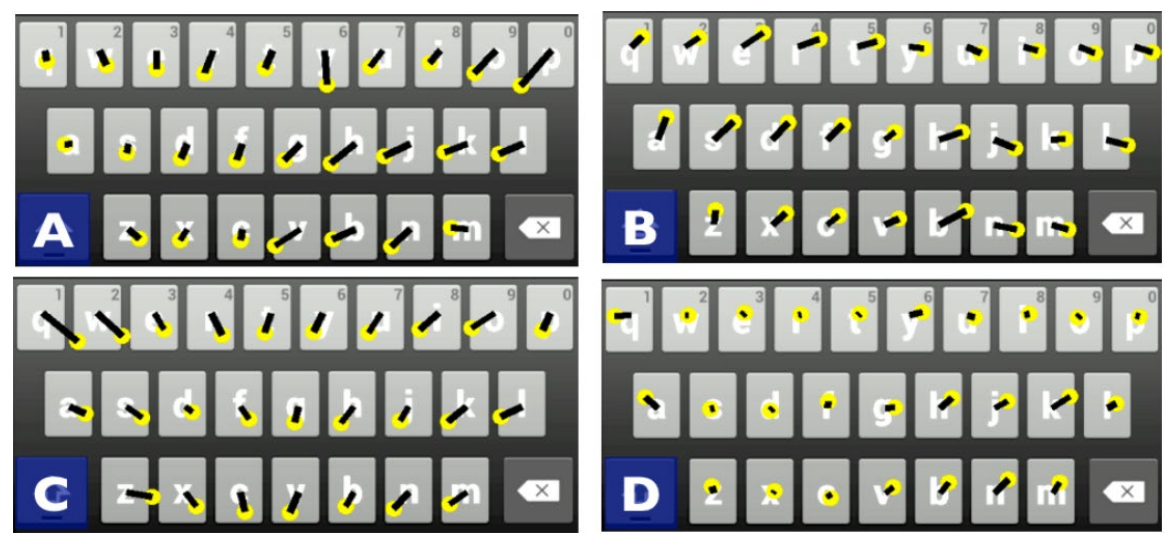

Figure 4. Tap offsets for each key for one participant. Yellow dots represent keypress centroids for each key, and black lines represent distances from visual key centers. (A) One-handed grip with left thumb typing. (B) One-handed grip with right thumb typing. (C) Two-handed grip with one index finger typing. (D) Two-handed grip with two thumbs typing. One can easily see how different grips result in different systematic keypress shifts. Figure used from prior work [11] with permission from the authors.

\subsection{SwitchBack (2015)}

Prior work has shown how divided people's attention becomes when using mobile devices on-the-go. In particular, work by Oulasvirta et al. [32,33] has shown that we interact in short few-second "bursts," repeatedly looking at and away from our mobile screens. When we look away from-and then return our gaze to- our device screens, we have to visually search to find where we previously left off. This visual search consumes precious time in what is already an overly short window of interaction time. To aid visual search and resumption from distraction, Markiakakis et al. [23] created SwitchBack, a system that uses a device's front-facing camera to track the user's eyes and face, and provide on-screen highlighting when the eyes return to the screen after looking away.

In our prototype, we applied SwitchBack to the problem of reading an article, looking away, and upon resuming, having to find one's place in the article where one left off. When facing a sea of text on a mobile screen, resuming reading can be particularly difficult. SwitchBack briefly highlighted the last line of text a user read when the user's eyes returned to the screen (see Figure 5).

Our formal evaluation of 17 participants had them stand still or walk on a treadmill while performing a reading comprehension task that, for some trials, was interrupted by a distraction task involving the identification of rotated "T" or "L" shapes in a mixed field of those letters [52]. Also, SwitchBack was enabled for some trials and not for others. Thus, the experiment was a $2 \times 2 \times 2$ within-subjects factorial design. The results showed that across all users, in the absence of distractions, reading speed and comprehension were the same with or without SwitchBack. But in the presence of distractions, reading speed was about 19 words per minute higher with SwitchBack than without, a statistically significant increase of $7.7 \%$. This benefit came without any cost to reading comprehension. 

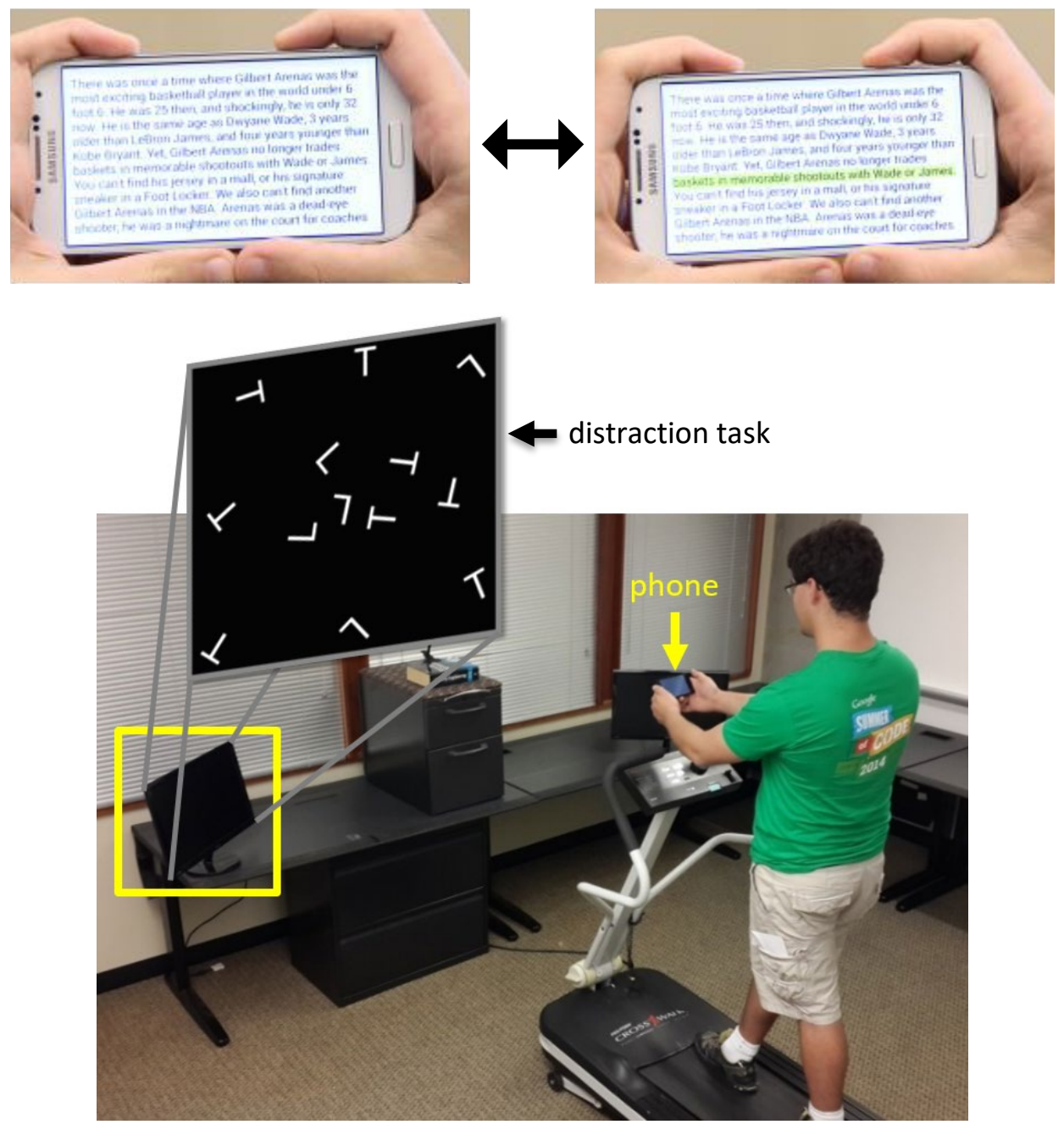

Figure 5. (top) SwitchBack highlights the last line of text read when the face and eyes return to the screen after looking away, aiding in task resumption. (bottom) Our experiment involving a participant walking on a treadmill, reading on a mobile device, and intermittently counting " $T$ " or " $L$ " shapes in a distraction task [52]. Figure adapted from prior work [23] with permission from the authors.

\subsection{Drunk User Interfaces (2018)}

All of the aforementioned projects followed a similar pattern, which was to sense a user's situation, model or interpret that user-situation system in some way, and then adapt, visually or "behind the scenes," such that the user's interaction with the system could be improved in light of their situation. This sense-model-adapt sequence is a powerful (and suitable) one for addressing SIIDs. However, some projects might focus only on a subset of this sequence. Such was the case with Drunk User Interfaces by Mariakakis et al. [24], which were interfaces that presented basic human performance tasks to users on their smartphones in order to measure their blood alcohol level (BAL). Plausible motivations for using a smartphone to unobtrusively measure BAL included discounts on car insurance for agreeing to self-monitor, self-imposed prevention from "drunk texting" [17], informal assessment by service providers like bartenders, and self-awareness and education about the effects of drinking on one's own BAL.

Our system of drunk user interfaces, or "DUIs," created user-specific models that could infer a person's BAL with surprising accuracy, specifically to within $0.005 \% \pm 0.007 \%{ }^{4}$ of a commercial breathalyzer. The

\footnotetext{
${ }^{4}$ This expression represents the mean error percentage plus-or-minus one standard deviation.
} 
five drunk user interfaces, or "DUIs," are shown in Figure 6. They were: (1) typing on a smartphone keyboard; (2) swiping a gesture password on a smartphone lock screen; (3) holding a smartphone flat with a finger covering the camera and flash, which enables photoplethysmographic readings of one's heart rate [16]; (4) tapping on a square when it changes color, for simple reaction time; and (5) tapping on one of four squares when it changes color, for choice reaction time.
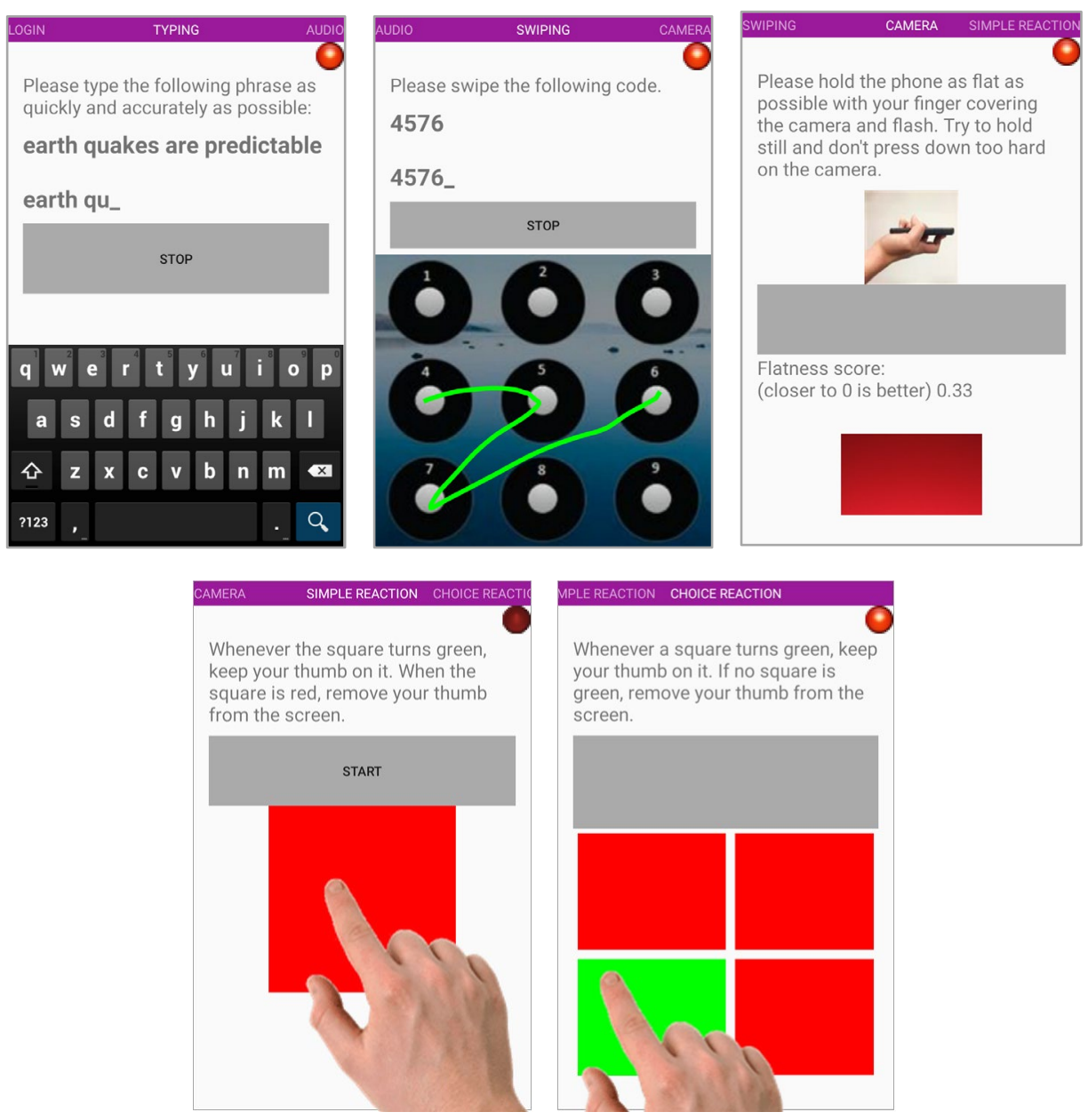

Figure 6. The drunk user interfaces, or DUIs, that were issued as performance tasks to users. By using random forest regression models on data from these tasks, we could predict a user's BAL to within $0.005 \% \pm 0.007 \%$ of the measures from a commercial breathalyzer, on average.

To build our predictive user models, we had each of 14 participants come to our lab each day for five consecutive days. During each session, they used the DUIs while sober and then after drinking vodka and orange juice such that their BAL, as measured by a commercial breathalyzer, climbed from $0.00 \%$ on the first day to $0.08 \%$ on the fifth day, at $+0.02 \%$ increments per day. ${ }^{5}$ Each DUI was briefly used five times for a total of four minutes of use for all DUIs. Then, we used random forest regression to build user-specific predictive models using various features from each DUI task. Although the features are too numerous to list here, they included things like mean distances from key centers while typing, mean speeds of gesture password segments, mean heart rates, and mean finger lift times on the reaction-time tasks. Using a decision

\footnotetext{
${ }^{5}$ The legal BAL limit for operating a motor vehicle in the United States is $0.08 \%$. A value under $0.04 \%$ is considered sober by the National Advisory Council on Alcohol Abuse and Alcoholism, while a value of $0.04 \%$ and above is considered intoxicated.
} 
threshold of $0.04 \%$ BAL for sober/intoxicated, our final DUI models had a sensitivity (true positive rate) of $93.9 \%$ and a specificity (true negative rate) of $82.3 \%$.

\subsection{RainCheck (2018)}

The projects described thus far address factors in the Behavioral, Attentional, and Affective sections of Table 1. RainCheck introduced a solution for an Environmental factor, namely rainwater. Specifically, with most capacitive-sensing touch screen smartphones, water on the screen interferes with finger touches, making such phones difficult or impossible to operate when wet. Rainwater causes all sorts of problems, from creating finger touches that do not occur, to splitting single touches into multiples, to shifting touches elsewhere (Figure 7).
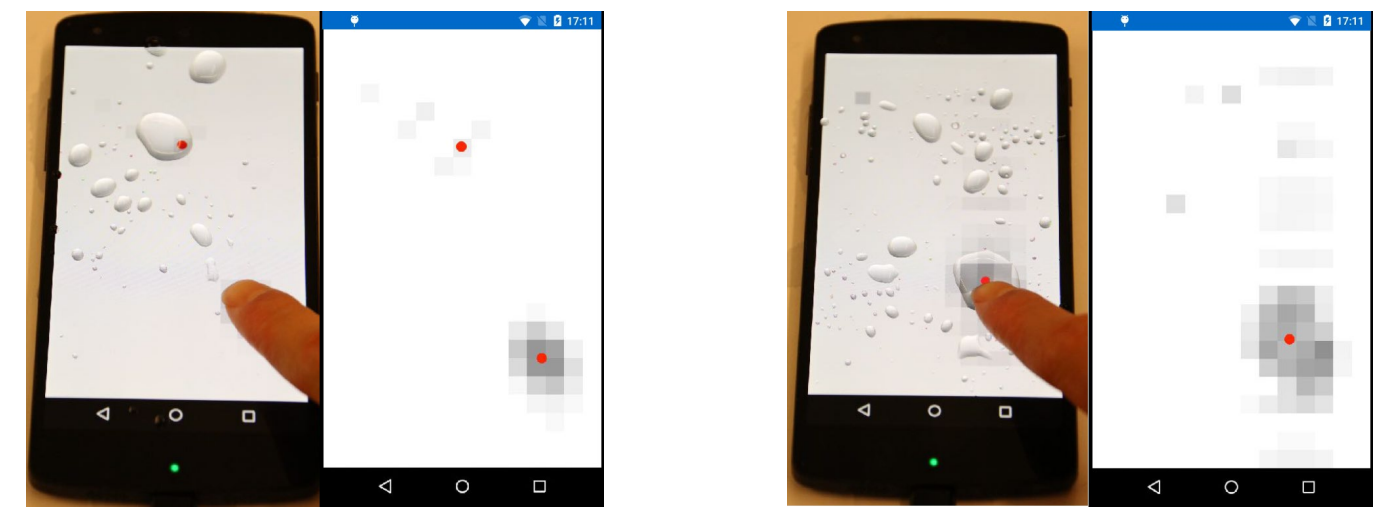

Figure 7. (left) One touch in the bottom-right corner of the screen is sensed as two "touches" due to a large water droplet in the upper-middle of the screen. (right) A touchpoint is shifted up considerably due to the touch occurring adjacent to a large water droplet.

For RainCheck, Tung et al. [43] devised an algorithm and an interaction technique to make regular capacitive-sensing touch screens operable when wet. RainCheck's capabilities were achieved purely through software without requiring any hardware modifications or add-ons. The RainCheck algorithm used heuristics to first filter out possible touches likely caused by water droplets. These heuristics used connected components over raw capacitance readings and looked at peaks, variances, and standard deviations of the sensor values, among other things. When the algorithm could not determine whether a possible touch was, in fact, a touch or a water droplet, RainCheck enabled marking menus [19] such that a finger could perform a swipe to resolve a target (see Figure 8). As swipes convey more information than taps, they were more reliable to accurately recognize, even in the presence of water droplets.

We conducted two studies of RainCheck. In the first, we evaluated the performance of our filtering algorithm with six gesture types in dry and wet conditions. The gestures were swipes in the four cardinal directions and pinch-to-zoom in and out. Overall, RainCheck resulted in a 75.7\% reduction in errors with water on the touch screen. In the second study, we evaluated RainCheck's ability to improve target selection. RainCheck reduced target selection time by $80.0 \%$ in the presence of water on the touch screen. It also improved touch accuracy by $47.9 \%$. In both studies, when the screen was dry, RainCheck had no detrimental effect on tap or swipe recognition, meaning that RainCheck could be enabled at all times without negative impacts. 

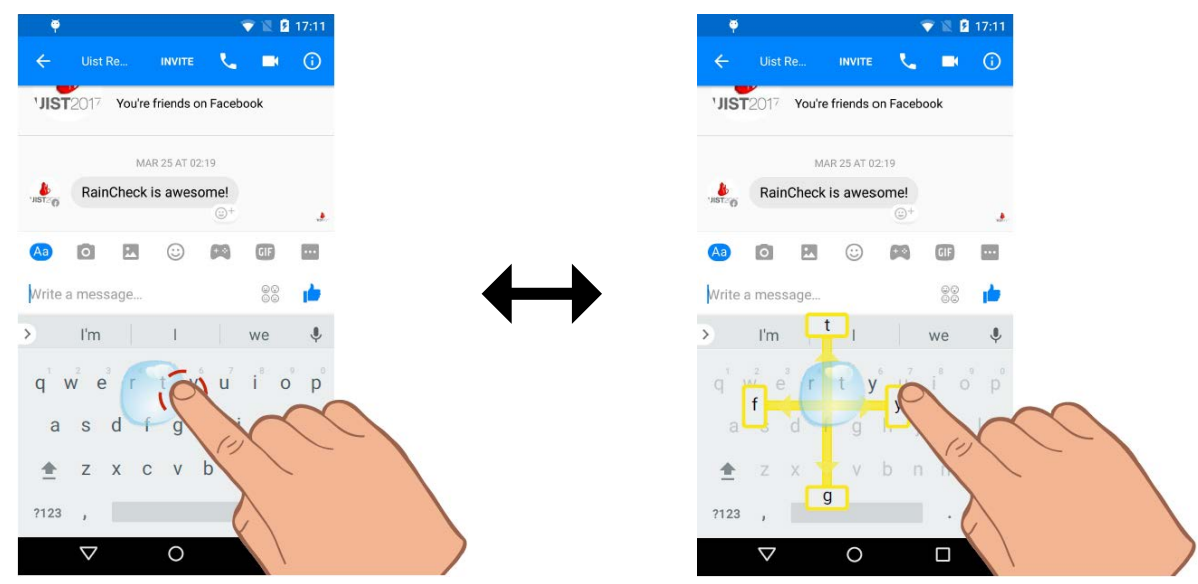

Figure 8. RainCheck looks for capacitive touch areas that do not conform to the usual shapes and sizes of typical finger touches. When it detects an unusual touch signature in an area dense with potential targets, it relegates those targets to a marking menu [19], allowing the user to quickly swipe over the desired target. Figure adapted from prior work [43] with permission from the authors.

\section{Open Challenges and Future Work: The Next Decade}

The seven projects above address various factors that cause situationally induced impairments and disabilities (SIIDs). Specifically, the factors addressed were walking, hand grips, divided attention, distraction, inebriation, and rainwater interference. But these factors represent only a small fraction of those shown in Table 1.

What would enable the widespread amelioration of SIIDs of many kinds? If software toolkits were made widely available that enabled mobile application developers to make "situationally aware mobile apps" by hooking into ready-made sensors, user models, and adaptive interface capabilities, it would no longer take tremendous developer effort and initiative to make custom apps that address SIIDs. Flexible toolkits with the right levels of abstraction could enable developers to address a range of factors from Table 1. To do so completely, it might require new hardware sensors or more advanced machine learning; but even without such advances, the range of sensors on board today's smartphones enable many of the factors in Table 1 to be addressed through software, as shown by the examples in this article.

We also must admit that we do not yet have a complete scientific understanding of the effects of many types of factors that cause SIIDs. Although some of these factors are straightforward and perhaps do not require formal scientific study, others do if we are to discover the best ways to sense and model situations, and adapt technologies to them. For example, in the Social category of Table 1, there are complex factors that require a deep understanding of human communication and social psychology. If we are to design situationally aware mobile devices and apps that can better support effective and edifying social exchanges among people, we must understand how those exchanges occur and how they should occur with technology in the mix.

Finally, we should recognize the ethical dilemmas raised by increasing the situational awareness of our mobile devices. More situationally aware mobile devices mean more devices permeating our lives that "know" more about us - about the situations, contexts, and environments we are in. For example, if we are to effectively address some of the Social factors in Table 1, our devices would need to recognize when we are in social situations, and perhaps with whom. Mobile devices already can be given permission to know our locations, but moving from location to situation might well be a step too far.

Another ethical dilemma concerns our design goals. The projects described in this article make it easier to operate mobile devices under the influence of SIIDs. But should we be making it easier to do so? Or should we be designing technologies to discourage people from interacting in situations that could be potentially dangerous to themselves or others? A particularly disconcerting example is whether we should make it easier for people to send text messages while driving, or whether we should create technologies to detect driving 
and prevent the sending of such messages. One response is the "safe sex" answer: people are going to use mobile devices in all kinds of situations anyway, so we might as well make devices as smart and safe as possible. But another response is the eudaimonic one-that we should create technologies that encourage people to behave as their very best selves, not given to momentary impulses [25].

\section{Conclusion}

Owing to their varied and pervasive contexts of use, today's mobile devices, especially but not limited to smartphones, do not currently support users' experiencing situationally induced impairments and disabilities (SIIDs) $[39,40]$. In this era of mobile and ubiquitous computing, the assumptions about users' abilities and contexts that underlie the use of desktop computing systems no longer apply. Unfortunately, our mobile devices are nearly oblivious to the situations, contexts, and environments in which they are used, and therefore present themselves as little more than shrunken desktops in thin casings.

This article has argued for more situationally aware mobile devices that can aid users by addressing SIIDs. It has also presented a categorized list of factors that can cause SIIDs (Table 1), and a two-dimensional space for characterizing impairments (Table 2). Seven research projects were offered as examples, all of which contained functional prototypes and formal quantitative evaluations. Many of these projects followed a sense-model-adapt design pattern suitable for addressing SIIDs.

Just as computing and people's relation to it have changed dramatically in the last decade, so too will the next decade take computing further from staid office environments and deeper into our homes, lives, and relationships. Let us make our mobile devices safer, smarter, and better integrated into our lives, while always remaining vigilant not to undermine what makes life worth living in the first place.

\section{ACKNOWLEDGMENTS}

The author thanks his collaborators on the projects mentioned in Section 4, especially Mayank Goel, Alex Mariakakis, and Shwetak Patel. This work was supported in part by Microsoft Research, Google, Intel, Baidu, the Mani Charitable Foundation, and the National Science Foundation under grant IIS-1217627. Any opinions, findings, conclusions, or recommendations expressed in this work are those of the author and do not necessarily reflect those of any particular supporter or collaborator.

\section{REFERENCES}

[1] Hamed Aleaziz. (2015). 'Heads up!' Hayward debuts snarky traffic signs. SFGate.com. https://www.sfgate.com/bayarea/article/Heads-up-Hayward-debuts-snarky-traffic-signs6079819.php\#photo-7522859

[2] Leon Barnard, Ji Soo Yi, Julie A. Jacko, and Andrew Sears. (2005). An empirical comparison of usein-motion evaluation scenarios for mobile computing devices. International Journal of HumanComputer Studies 62 (4), 487-520. DOI:10.1016/j.ijhcs.2004.12.002

[3] Joanna Bergstrom-Lehtovirta, Antti Oulasvirta, and Stephen Brewster. (2011). The effects of walking speed on target acquisition on a touchscreen interface. Proceedings of the ACM Conference on Human-Computer Interaction with Mobile Devices and Services (MobileHCI '11). New York: ACM Press, 143-146. DOI:10.1145/2037373.2037396

[4] Frank Bowe. (1987). Making computers accessible to disabled people. M.I.T. Technology Review 90, $52-59,72$.

[5] Andrew Bragdon, Eugene Nelson, Yang Li, and Ken Hinckley. (2011). Experimental analysis of touch-screen gesture designs in mobile environments. Proceedings of the ACM Conference on Human Factors in Computing Systems (CHI '11). New York: ACM Press, 403-412. DOI:10.1145/1978942.1979000

[6] Jane E. Brody. (2015). Not just drivers driven to distraction. The New York Times, D5. https://well.blogs.nytimes.com/2015/12/07/its-not-just-drivers-being-driven-to-distraction/

[7] Alan Chamberlain and Roy Kalawsky. (2004). A comparative investigation into two pointing systems for use with wearable computers while mobile. Proceedings of the IEEE International Symposium on Wearable Computers (ISWC '04). Washington, D.C.: IEEE Computer Society, 110-117. DOI:10.1109/ISWC.2004.1

[8] Ericka Chickowski. (2004). It’s all about access. Alaska Airlines Magazine 28, 26-31, 80-82. 
[9] Krzysztof Z. Gajos, Amy Hurst, and Leah Findlater. (2012). Personalized dynamic accessibility. Interactions 19 (2), 69-73. DOI:10.1145/2090150.2090167

[10] Mayank Goel, Leah Findlater, and Jacob O. Wobbrock. (2012). WalkType: Using accelerometer data to accommodate situational impairments in mobile touch screen text entry. Proceedings of the ACM Conference on Human Factors in Computing Systems (CHI' 12). New York: ACM Press, 2687-2696. DOI:10.1145/2207676.2208662

[11] Mayank Goel, Alex Jansen, Travis Mandel, Shwetak N. Patel, and Jacob O. Wobbrock. (2013). ContextType: Using hand posture information to improve mobile touch screen text entry. Proceedings of the ACM Conference on Human Factors in Computing Systems (CHI '13). New York: ACM Press, 2795-2798. DOI:10.1145/2470654.2481386

[12] Mayank Goel, Jacob O. Wobbrock, and Shwetak N. Patel. (2012). GripSense: Using built-in sensors to detect hand posture and pressure on commodity mobile phones. Proceedings of the ACM Symposium on User Interface Software and Technology (UIST '12). New York: ACM Press, 545-554. DOI:10.1145/2380116.2380184

[13] Chris Graham. (2016). Road signs warn pedestrians not to use smartphones. The Telegraph. https://www.telegraph.co.uk/news/worldnews/europe/sweden/12139462/Road-signs-warn-pedestriansnot-to-use-smartphones.html

[14] Asela Gunawardana, Tim Paek, and Christopher Meek. (2010). Usability guided key-target resizing for soft keyboards. Proceedings of the ACM Conference on Intelligent User Interfaces (IUI '10). New York: ACM Press, 111-118. DOI:10.1145/1719970.1719986

[15] Clyde Haberman. (2018). The dangers of walking while texting. The New York Times, SR10. https://www.nytimes.com/2018/03/17/opinion/do-not-read-this-editorial-while-walking.html

[16] Teng Han, Xiang Xiao, Lanfei Shi, John Canny, and Jingtao Wang. (2015). Balancing accuracy and fun: Designing camera based mobile games for implicit heart rate monitoring. Proceedings of the ACM Conference on Human Factors in Computing Systems (CHI '15). New York: ACM Press, 847856. DOI:10.1145/2702123.2702502

[17] Aditya Joshi, Abhijit Mishra, Balamurali AR, Pushpak Bhattacharyya, and Mark James Carman. (2015). A computational approach for automatic prediction of drunk-texting. Proceedings of the 53rd Annual Meeting of the Association for Computational Linguistics and the 7th International Joint Conference on Natural Language Processing of the Asian Federation of Natural Language Processing (ACL-IJCNLP '15). Cambridge, MA: Association for Computational Linguistics. https://arxiv.org/abs/1610.00879

[18] Shaun K. Kane, Jacob O. Wobbrock, and Ian E. Smith. (2008). Getting off the treadmill: Evaluating walking user interfaces for mobile devices in public spaces. Proceedings of the ACM Conference on Human-Computer Interaction with Mobile Devices and Services (MobileHCI '08). New York: ACM Press, 109-118. DOI:10.1145/1409240.1409253

[19] Gordon Kurtenbach and W. Buxton. (1994). User learning and performance with marking menus. Proceedings of the ACM Conference on Human Factors in Computing Systems (CHI '94). New York: ACM Press, 258-264. DOI:10.1145/191666.191759

[20] Min Lin, Rich Goldman, Kathleen J. Price, Andrew Sears, and Julie Jacko. (2007). How do people tap when walking? An empirical investigation of nomadic data entry. International Journal of HumanComputer Studies 65 (9), 759-769. DOI:10.1016/j.jhhcs.2007.04.001

[21] Min Lin, Kathleen J. Price, R. Goldman, Andrew Sears, and Julie A. Jacko. (2005). Tapping on the move-Fitts' law under mobile conditions. Proceedings of the 16th Annual Information Resources Management Association International Conference (IRMA '05). Hershey, PA: Idea Group, 132-135. DOI:10.4018/978-1-59140-822-2.ch032

[22] Jennifer Mankoff, Gillian R. Hayes, and Devva Kasnitz. (2010). Disability studies as a source of critical inquiry for the field of assistive technology. Proceedings of the ACM SIGACCESS Conference on Computers and Accessibility (ASSETS '10). New York: ACM Press, 3-10. DOI:10.1145/1878803.1878807

[23] Alex Mariakakis, Mayank Goel, Md. Tanvir Islam Aumi, Shwetak N. Patel, and Jacob O. Wobbrock. (2015). SwitchBack: Using focus and saccade tracking to guide users' attention for mobile task resumption. Proceedings of the ACM Conference on Human Factors in Computing Systems (CHI '15). New York: ACM Press, 2953-2962. DOI:10.1145/2702123.2702539

[24] Alex Mariakakis, Sayna Parsi, Shwetak N. Patel, and Jacob O. Wobbrock. (2018). Drunk User Interfaces: Determining blood alcohol level through everyday smartphone tasks. Proceedings of the 
ACM Conference on Human Factors in Computing Systems (CHI '18). New York: ACM Press. Paper No. 234. DOI:10.1145/3173574.3173808

[25] Elisa D. Mekler and Kasper Hornbæk. (2016). Momentary pleasure or lasting meaning?

Distinguishing eudaimonic and hedonic user experiences. Proceedings of the ACM Conference on Human Factors in Computing Systems (CHI '16). New York: ACM Press, 4509-4520. DOI:10.1145/2858036.2858225

[26] Sachi Mizobuchi, Mark Chignell, and David Newton. (2005). Mobile text entry: Relationship between walking speed and text input task difficulty. Proceedings of ACM Conference on Human-Computer Interaction with Mobile Devices and Services (MobileHCI '05). New York: ACM Press, 122-128. DOI:10.1145/1085777.1085798

[27] Tanya Mohn. (2017). Reading this while walking? In Honolulu, it could cost you. The New York Times, B1. https://www.nytimes.com/2017/10/23/business/honolulu-walking-and-texting-fine.html

[28] Terhi Mustonen, Maria Olkkonen, and Jukka Häkkinen. (2004). Examining mobile phone text legibility while walking. Extended Abstracts of the ACM Conference on Human Factors in Computing Systems (CHI '04). New York: ACM Press, 1243-1246. DOI:10.1145/985921.986034

[29] Alan F. Newell. (1995). Extra-ordinary human-computer interaction. In Extra-Ordinary HumanComputer Interaction: Interfaces for Users with Disabilities, Alistair D. N. Edwards (ed.). Cambridge, England: Cambridge University Press, 3-18.

[30] Rick Noack. (2014). A Chinese city is asking smartphone users to walk in their own sidewalk lane. The Washington Post. https://www.washingtonpost.com/news/worldviews/wp/2014/09/15/a-chinesecity-is-asking-smartphone-users-to-walk-in-their-own-sidewalk-lane/

[31] Rick Noack. (2016). This city embedded traffic lights in the sidewalks so that smartphone users don't have to look up. The Washington Post.

https://www.washingtonpost.com/news/worldviews/wp/2016/04/25/this-city-embedded-traffic-lightsin-the-sidewalks-so-that-smartphone-users-dont-have-to-look-up/

[32] Antti Oulasvirta. (2005). The fragmentation of attention in mobile interaction, and what to do with it. Interactions 12 (6), 16-18. DOI:10.1145/1096554.1096555

[33] Antti Oulasvirta, Sakari Tamminen, Virpi Roto, and Jaana Kuorelahti. (2005). Interaction in 4-second bursts: The fragmented nature of attentional resources in mobile HCI. Proceedings of the ACM Conference on Human Factors in Computing Systems (CHI '05). New York: ACM Press, 919-928. DOI:10.1145/1054972.1055101

[34] Lucy Pasha-Robinson. (2017). Honolulu bans texting and walking with "distracted pedestrian law." The Independent. https://www.independent.co.uk/news/world/americas/honolulu-texting-walkinghawaii-city-distracted-pedestrian-law-a $8018686 . \mathrm{html}$

[35] Allison Ruby Reid-Cunningham. (2009). Anthropological theories of disability. Journal of Human Behavior in the Social Environment 19 (1), 99-111. DOI:10.1080/10911350802631644

[36] Matt Richtel. (2010). Forget gum. Walking and using phone is risky. The New York Times, A1. http://www.nytimes.com/2010/01/17/technology/17distracted.html

[37] Martin Schedlbauer and Jesse Heines. (2007). Selecting while walking: An investigation of aiming performance in a mobile work context. Proceedings of the 13th Americas Conference on Information Systems (AMCIS '07). Atlanta, GA: Association for Information Systems. http://citeseerx.ist.psu.edu/viewdoc/summary?doi=10.1.1.102.935

[38] Bastian Schildbach and Enrico Rukzio. (2010). Investigating selection and reading performance on a mobile phone while walking. Proceedings of the ACM Conference on Human-Computer Interaction with Mobile Devices and Services (MobileHCI '10). New York: ACM Press, 93-102. DOI:10.1145/1851600.1851619

[39] Andrew Sears, Min Lin, Julie Jacko, and Yan Xiao. (2003). When computers fade... Pervasive computing and situationally-induced impairments and disabilities. Proceedings of the 10th International Conference on Human-Computer Interaction (HCI International '03). Mahwah, NJ: Lawrence Erlbaum, 1298-1302.

[40] Andrew Sears and Mark Young. (2003). Physical disabilities and computing technologies: An analysis of impairments. In The Human-Computer Interaction Handbook (1st ed.), Julie A. Jacko and Andrew Sears (eds.). Hillsdale, NJ: Lawrence Erlbaum, 482-503. http://dl.acm.org/citation.cfm?id=772105

[41] Jim Sinclair. (2010). Cultural commentary: Being autistic together. Disability Studies Quarterly 30 (1). http://www.dsq-sds.org/article/view/1075/1248 
[42] Leah L. Thompson, Frederick P. Rivara, Rajiv C. Ayyagari, and Beth E. Ebel. (2013). Impact of social and technological distraction on pedestrian crossing behaviour: An observational study. Injury Prevention 19 (4), 232-237. DOI:10.1136/injuryprev-2012-040601

[43] Ying-Chao Tung, Mayank Goel, Isaac Zinda, and Jacob O. Wobbrock. (2018). RainCheck: Overcoming capacitive interference caused by rainwater on smartphones. Proceedings of the ACM International Conference on Multimodal Interfaces (ICMI '18). New York: ACM Press, 464-471. DOI:10.1145/3242969.3243028

[44] Kristin Vadas, Nirmal Patel, Kent Lyons, Thad Starner, and Julie Jacko. (2006). Reading on-the-go: A comparison of audio and hand-held displays. Proceedings of the ACM Conference on HumanComputer Interaction with Mobile Devices and Services (MobileHCI '06). New York: ACM Press, 219-226. DOI:10.1145/1152215.1152262

[45] Gregg C. Vanderheiden. (1997). Anywhere, anytime (+anyone) access to the next-generation WWW. Computer Networks and ISDN Systems 29 (8-13), 1439-1446. DOI:10.1016/S0169-7552(97)00067-6

[46] A. J. Vicens. (2014). Texting while walking is obviously dumb. So why can't we stop doing it? Mother Jones. https://www.motherjones.com/environment/2014/12/texting-walking-injuries-deaths/

[47] Jacob O. Wobbrock. (2006). The future of mobile device research in HCI. In ACM CHI 2006 Workshop on "What is the Next Generation of Human-Computer Interaction?", 131-134.

[48] Jacob O. Wobbrock. (2019). Situationally-induced impairments and disabilities. In Web Accessibility (2nd ed.), Yeliz Yesilada and Simon Harper (eds.). London, England: Springer-Verlag. To appear. DOI:10.1007/978-1-4471-7440-0_5

[49] Jacob O. Wobbrock, Krzysztof Z. Gajos, Shaun K. Kane, and Gregg C. Vanderheiden. (2018). Abilitybased design. Communications of the ACM 61 (6), 62-71. DOI:10.1145/3148051

[50] Jacob O. Wobbrock, Shaun K. Kane, Krzysztof Z. Gajos, Susumu Harada, and Jon Froehlich. (2011). Ability-based design: Concept, principles, and examples. ACM Transactions on Accessible Computing 3 (3). Article No. 9. DOI:10.1145/1952383.1952384

[51] World Health Organization. (2000). International Classification of Impairments, Disabilities, and Handicaps. Geneva, Switzerland: World Health Organization.

[52] Takemasa Yokoyama, Hiroki Sakai, Yasuki Noguchi, and Shinichi Kita. (2014). Perception of direct gaze does not require focus of attention. Scientific Reports 4 (3858), 1-7. DOI:10.1038/srep03858 